OPEN ACCESS

Edited by:

Ticiana A. Leal,

University of Wisconsin-Madison,

United States

Reviewed by:

Jinbo Yue,

Shandong University, China

Wan Xiangbo,

Six Affiliated Hospital of Sun

Yat-sen University, China

Huilan Zhang,

Huazhong University of Science and Technology, China

*Correspondence:

Weidong Han

hanwd@zju.edu.cn

Hongming Pan

panhongming@zju.edu.cn

Qin Pan

tianpanqin@yeah.net

${ }^{\dagger}$ These authors have contributed equally to this work

Specialty section:

This article was submitted to

Thoracic Oncology,

a section of the journal

Frontiers in Oncology

Received: 19 October 2020 Accepted: 30 December 2020 Published: 17 February 2021

Citation:

Zhai C, Zhang $X$, Ren L, You L, Pan Q, Pan H and Han W (2021) The

Efficacy and Safety of Anlotinib Combined With PD-1 Antibody for

Third-Line or Further-Line Treatment of Patients With Advanced Non-Small-Cell Lung Cancer.

Front. Oncol. 10:619010. doi: 10.3389/fonc.2020.619010

\section{The Efficacy and Safety of Anlotinib Combined With PD-1 Antibody for Third-Line or Further-Line Treatment of Patients With Advanced Non- Small-Cell Lung Cancer}

\author{
Chongya Zhai ${ }^{\dagger}$, Xiaoling Zhang ${ }^{\dagger}$, Lulu Ren, Liangkun You, Qin Pan ${ }^{*}$, Hongming Pan * \\ and Weidong Han* \\ Department of Medical Oncology, Sir Run Run Shaw Hospital, College of Medicine, Zhejiang University, Hangzhou, China
}

Background: Both anlotinib and programmed death 1 (PD-1) monoclonal antibody $(\mathrm{mAb})$ have been approved for the third line treatment of metastatic non-small cell lung cancer (NSCLC). However, the combination of these two standard therapies has not been investigated in third-line or further-line treatment of patients with advanced NSCLC.

Methods: We reviewed 22 patients with NSCLC who received anlotinib combined with PD-1 mAb therapy from July 2018 to October 2019 at Sir Run Run Shaw Hospital. Based on the baseline characteristics, PD-L1 expression and EGFR mutation status, we retrospectively analyzed the efficacy and safety of this combination therapy by RESIST 1.1 and CTCAE 5.0.

Results: The combination treatment of anlotinib and PD-1 mAb in 22 NSCLC patients gained a median PFS of 6.8 months and a median OS of 17.3 months. The disease control rate (DCR) was $90.9 \%$, and the objective response rate (ORR) was $36.4 \%$, where $1(4.6 \%)$ patient achieved complete response (CR) and 7 (31.8\%) patients achieved partial response (PR). The median time to response was 3.9 months, and the median duration of the response was 6.8 months. The common grades 1-2 adverse events were fatigue 10/22 (45.5\%), decreased appetite 9/22 (40.9\%), hypertension 10/22 (45.5\%); the common grades 3-4 adverse events were hypertension 2/22 (9.1\%) and mouth ulceration 2/22 (9.1\%).

Conclusion: Anlotinib combined with PD-1 mAb showed promising efficacy in third-line or further-line treatment of NSCLC, and its adverse effects is tolerable.

Keywords: NSCLC, anlotinib hydrochloride, third line of therapy, TP53, EGFR

\section{INTRODUCTION}

Lung cancer is the leading cause of cancer-related morbidity and mortality, non-small-cell lung cancer (NSCLC) is the most prevalent subtype with a poor prognosis owing to the presence of locally advanced or wide metastasis in the majority of patients at the time of diagnosis or postoperative recurrence (1). Significant progress has been made in the treatment of advanced 
NSCLC in the past 10 years. In patients with positive drive mutation, the drugs represented by EGFR-TKI achieved nearly 3-year overall survival (2). For patients with negative drive mutation, PD-1 mAb can significantly improve the therapeutic efficacy and prolong the overall survival (3). In the first-line treatment, it can be used alone or combined with chemotherapy, while the second-line treatment is recommended to use PD-1 $\mathrm{mAb}$ alone (4). When PD-1 mAb is used alone, the overall response rate and PFS are not satisfactory (5-7). Some patients even suffered from hyper-progression with single immunotherapy due to high metastatic burden (8).

Anlotinib is a multi-target drug approved for the third line treatment of advanced NSCLC, which could inhibit the vascular endothelial growth factor (VEGFR) 1-3, platelet-derived growth factor (PDGFR) $\alpha$, PDGFR $\beta$, C-proto-oncogenic receptor tyrosine kinase (C-KIT) and RET. It represses the tumor angiogenesis by down-regulating major pro-angiogenic factors, such as VEGF, PDGF-BB and fibroblast growth factor (FGF). Surprisingly, anlotinib used in the third line treatment of NSCLC gained a median PFS of 5.4 months (9). Interestingly, one study has shown that anlotinib could enhance the ratio of $\mathrm{CD} 8 / \mathrm{FoxP}^{+}$ $\mathrm{T}$ cell in tumor tissues thus altering the tumor microenvironment (10). Moreover, anlotinib was proved to promote the infiltration of natural killer (NK) cells, M1-like tumor-associated macrophage (TAM) and dendritic cells in lung cancer mouse model, and the combination of anlotinib with immune checkpoint inhibitor gained better therapeutic response (11). These results suggest that anlotinib is involved in the regulation of tumor immune microenvironment, and the combination therapy with PD-1 mAb may be the future exploration direction.

There have been many reports on the treatment of NSCLC with PD-1 mAb combined with anti-angiogenetic agents. In patients with disease progression after first-line treatment, Sintilimab combined with bevacizumab showed unexpected efficiency. The combination therapy achieved a median PFS of 6 months, and the patients were well tolerated (12). Besides, Sintilimab combined with anlotinib in 22 patients of NSCLC at first line treatment gained an ORR of $68.2 \%$ and DCR of $100 \%, 2$ grade 3 treatment-related adverse events (TRAEs) occurred with no grade 4/5 observation, however, the PFS and OS was not available due to the short follow-up time (13). Taken together, Angiogenesis inhibitors combined with immune check point inhibitors in the treatment of advanced lung cancer has achieved preliminary clinical validation.

Anti-angiogenesis agents and PD-1 mAb can cooperate to alter the microenvironment of the tumor. The expression of PD-1 was up-regulated in relapsed tumor after the anti-VEGFR2 agent treatment (14). Studies have shown that PD-1 mAb and anti-VEGFR2 agent combination treatment could promote tumor vessel normalization and induce high endothelial venules (HEVs), which promoted lymphocyte infiltration and activity through the activation of lymphotoxin $\beta$ receptor (LT $\beta R$ ) signaling (15). In addition, anti-angiogenesis therapy could restore the response of effector $\mathrm{T}$ lymphocytes by breaking the tumor vessel barrier, and subsequent anti-PD-1 treatment further improved the activity of $\mathrm{T}$ lymphocytes, thereby synergistically leading to tumor shrinkage (16).
In this real-world evidence-based retrospective clinical study, we analyzed the efficacy and safety of anlotinib combined with PD-1 mAb for the third-line or further-line treatment of NSCLC patients. The results show that it has a good clinical application prospect and is worthy of further study.

\section{METHODS}

\section{Data Source}

We reviewed the records of a prospectively collected database of 22 patients with NSCLC who received anlotinib combined with PD-1 mAb, including sintilimab, nivolumab, pembrolizumab, toripalimab, and camrelizumab for the third-line or further treatment over the period of 1 year from July 2018 to October 2019 at Sir Run Run Shaw Hospital, College of Medicine, Zhejiang University, Hangzhou, China.

We retrospectively analyzed the baseline characteristics, PD-L1 expression, epithelial growth factor (EGFR) mutation status and prior and later treatment lines. All data were obtained by followup visits, telephone, electronic medical records, and letters. This study was approved by the Ethics Committee of Sir Run Run Shaw Hospital.

\section{Patient Selection}

The target samples included patients who received anlotinib and PD-1 mAb at Sir Run Run Shaw Hospital from July 2018 to October 2019. A definite histological or cytological diagnosis was required for the patients with NSCLC. The expected survival time was more than 3 months, and normal hematopoietic, hepatic, and renal function were prerequisite for enrollment. Exclusion criteria included patients with a history of autoimmune diseases or patients treated with steroids at a dose equivalent to or more than $10 \mathrm{mg}$ prednisone daily or other immunosuppressive drugs. Patients with central squamous cell carcinoma were also excluded. The selection flow chart was shown in the Figure 1.

\section{Study Variables}

The clinical response to anti-PD-1 mAb combined with anlotinib treatment was evaluated according to the Response Evaluation Criteria in Solid Tumors (RECIST) version 1.1. Every 2 or 3 cycles after the combined therapy was established, and shortterm efficacy was evaluated. The percentage of patients having achieved a complete response (CR: the disappearance of all target lesions) plus partial response (PR: at least a 30\% reduction in the sum of the diameters of the target lesions) recorded in the medical system were defined as the objective response rate (ORR). At least a $20 \%$ increase in the sum of the diameters of the target lesions were evaluated as PD, the lesions cannot be classified as PR or PD was evaluated as SD (stable disease). The percentage of patients with $\mathrm{CR}, \mathrm{PR}$ or $\mathrm{SD}$ was defined as the disease control rate (DCR). PFS was calculated as the time from the initiation of treatment with anti-PD-1 mAb combined with anlotinib therapy to progressive disease (PD) or death. OS referred to the time from the start of combination treatment to death from any cause. 
26 NSCLC patients treated with anlotinib and PD-1 mAb from July 2018 to October 2019

3 patients treated for first-line or second-line

23 patients were eligible

1 patient had no available follow-up data

22 patients for further analysis

FIGURE 1 | The patients selection flow chart.

\section{Statistical Analysis}

Descriptive statistics (percentages, means, medians) were used to describe baseline characteristics and clinical features of the sample of patients with NSCLC. ORR, PFS and OS were calculated to analyze the efficacy and clinical features of the patients treated with combination therapy. Statistical analyses were performed using SPSS statistical software (version 20.0; SPSS, IBM Corporation).

\section{RESULTS}

From July 2018 to October 2019, 22 patients with NSCLC were enrolled in this real-world study. Baseline demographics and clinical characteristics were exhibited in Table 1. In the final eligible sample, the median age of the patients was 65 years. Of $22,10(45.4 \%)$ patients were current or former smokers; 11 (50\%), never smokers; and 1 (4.6\%), unknown smoking status. Notably, 2 (9.1\%) patients were with ECOG status of 0, and 20 (90.9\%) patients had ECOG status of 1 at the time of diagnosis. $10(45.4 \%)$ patients were current or former smokers, and 11 (50\%) patients were never smoker. The smoking status was unknown in 1 (4.6\%) patient. Patients with non-squamous histology predominated: $15(68.2 \%)$ had adenocarcinoma, seven $(31.8 \%)$ had squamous cell carcinoma. Fourteen (63.6\%) patients had metastasis organ number more than 3 and eight (36.4\%) patients had metastasis organ number below 3. As for the PD-1 mAb treatment, a numbers of patients received nivolumab, pembrolizumab, Sintilimab, toripalimab, or camrelizumab treatment were 6 (27.3\%), 2 (9.1\%), 5 (22.7\%), 5 (22.7\%) and 4 (18.2\%), respectively. In addition, two (9.1\%) patients had PD-L1 tumor proportion score (TPS) above 50\%; seven $(31.8 \%)$, below $1 \%$; nine (40.9\%), between $1 \%$ and $49 \%$; four (18.2\%), unknown PD-L1 TPS. The efficacy of PD-1 mAb was related to the basic EGFR mutation status. In this study, 12 (54.6\%) patients had negative EGFR mutation status, followed by unknown EGFR mutation status (7, 31.8\%), positive EGFR mutation (3, 13.6\%), EGFR exon19 mutation (2, 9.1\%), and EGFR L858/T790M mutation (1, 4.5\%). 7 (31.8\%) patients had radiotherapy previously and $3(13.6 \%)$ patients ever received target therapy. The patients receiving $12 \mathrm{mg}$ or $10 \mathrm{mg}$ anlotinib dose numbered $6(27.3 \%)$ and $16(72.7 \%)$, respectively. The anlotinib dose was given after fully assessment of the tolerance and other basic physical status of the patients. The detailed anlotinib dose and treatment lines were listed in the Supplementary Table 1.

Patients response to anlotinib combined with PD-1 mAb was displayed in the Table 2. One (4.6\%) patient got CR; seven (31.8\%) patients got PR; 12 (54.5\%) patients remained SD; and two $(9.1 \%)$ patients had disease progressed. The ORR was $36.4 \%$ and the DCR was $90.9 \%$. The median time to response was 3.9 months, and the median duration of the response was 6.8 months. The median PFS (Figure 2A) of the combination therapy was 6.8 months (95\%CI: 3.4, 9.8), and the median OS (Figure 2B) of the treatment was 17.3 months (95\%CI: 16.1, 18.5). For each patient, the percent change in the sum of the longest diameter of target lesions diameter from the baseline was graphed in a waterfall plot (based on the treatment lines and patient's response, Figures 3A, B) and spider plot (Figure 4). For patients with brain metastasis subgroup analysis, six patients had brain metastasis, the median PFS was 4.7 months (95\% CI:2.37.1 months), 16 patients had no brain metastasis, the median PFS was 10.5 months (95\%CI:6.8-14.3 months), the $p$ value is 0.053 between two groups using the log-rank survival analysis.

The adverse events during the combination treatment were listed in the Table 3 . The most common grade 1-2 TRAEs were fatigue 10/22 (45.5\%), decreased appetite 9/22 (40.9\%), and hypertension $10 / 22(45.5 \%)$. The less common mild adverse events were nausea $3 / 22(13.6 \%)$, cough $2 / 22(9.1 \%)$ and hepatic function abnormal $3 / 22(13.6 \%)$. The grades $3-4$ adverse events were rash $2 / 22$ (9.1\%), hypertension $2 / 22$ (9.1\%), diarrhea $1 / 22(4.6 \%)$, mouth ulceration $2 / 22(9.1 \%)$, and pneumonitis $2 / 22(4.6 \%)$. 
TABLE 1 | Population characteristics.

\begin{tabular}{|c|c|}
\hline Baseline Characteristics & All patients $(n=22)$ \\
\hline \multicolumn{2}{|l|}{ Age } \\
\hline Median(range), years & $65(46-82)$ \\
\hline \multicolumn{2}{|l|}{ Gender, $\mathrm{n}(\%)$} \\
\hline Male & $14(63.6)$ \\
\hline Female & 8 (36.4) \\
\hline \multicolumn{2}{|c|}{ ECOG score at the time of diagnosis, n(\%) } \\
\hline 0 & $2(9.1)$ \\
\hline 1 & $20(90.9)$ \\
\hline \multicolumn{2}{|l|}{ Histological subtype, n(\%) } \\
\hline Adenocarcinoma & $15(68.2)$ \\
\hline Squamous cell carcinoma & 7 (31.8) \\
\hline \multicolumn{2}{|l|}{ Smoking status, $\mathrm{n}(\%)$} \\
\hline Never smoked & $11(50.0)$ \\
\hline Current or former smoker & $10(45.5)$ \\
\hline Unknown & $1(4.5)$ \\
\hline \multicolumn{2}{|c|}{ No.of prior systemic regimens, n(\%) } \\
\hline 2 & $15(68.2)$ \\
\hline 3 & $4(18.2)$ \\
\hline$\geq 4$ & $3(13.6)$ \\
\hline \multicolumn{2}{|c|}{ No.of organs of metastasis, n(\%) } \\
\hline$\geq 3$ & $14(63.6)$ \\
\hline$<3$ & $8(36.4)$ \\
\hline \multicolumn{2}{|l|}{ Anlotinib treatment dose, n(\%) } \\
\hline $12 \mathrm{mg}$ & $6(27.3)$ \\
\hline $10 \mathrm{mg}$ & $16(72.7)$ \\
\hline \multicolumn{2}{|l|}{ Anti-PD-1 mAbs, n(\%) } \\
\hline Nivolumab & $6(27.3)$ \\
\hline Pembrolizumab & $2(9.1)$ \\
\hline Sintilimab & $5(22.7)$ \\
\hline Toripalimab & $5(22.7)$ \\
\hline Camrelizumab & $4(18.2)$ \\
\hline \multicolumn{2}{|l|}{ PD-L1 TPS, n(\%) } \\
\hline$\geq 50 \%$ & $2(9.1)$ \\
\hline $1-49 \%$ & 9 (40.9) \\
\hline$<1 \%$ & $7(31.8)$ \\
\hline Unknown & $4(18.2)$ \\
\hline \multicolumn{2}{|l|}{ EGFR mutation status, n(\%) } \\
\hline Negative & $12(54.6)$ \\
\hline Positive & $3(13.6)$ \\
\hline Unknown & $7(31.8)$ \\
\hline \multicolumn{2}{|l|}{ Chemotherapy regimens, $\mathrm{n}(\%)$} \\
\hline 1 & $5(22.7)$ \\
\hline 2 & $11(50.0)$ \\
\hline$\geq 3$ & $6(27.3)$ \\
\hline \multicolumn{2}{|c|}{ Previous radiotherapy treatment, $\mathrm{n}(\%)$} \\
\hline No & $15(68.2)$ \\
\hline Yes & 7 (31.8) \\
\hline \multicolumn{2}{|l|}{ Previous target treatment, n(\%) } \\
\hline No & $19(86.4)$ \\
\hline Yes & $3(13.6)$ \\
\hline
\end{tabular}

Anti-PD-1, anti-programmed death-1; EGFR, epidermal growth factor receptor; ECOG, Eastern Cooperative Oncology Group; PD-L1, programmed death ligand-1; TPS, tumor proportion score.

\section{DISCUSSION}

From the retrospective summary of the anlotinib combined with the PD-1 mAb treatment, the efficacy was promising and unexpected. The third-line treatment of sole anlotinib in NSCLC gained a median PFS of 5.4 months and the median OS of 9.6 months (9), whereas combination treatment exhibited a median PFS of 6.8 months, median OS of 17.3 months and the ORR of $36.4 \%$, providing an
TABLE 2 | Efficacy of Anlotinib combined with Anti-PD-1 mAbs in third-line or further treatment of NSCLC patients.

\begin{tabular}{lc}
\hline Efficacy & All patients $\mathbf{( n = 2 2 )}$ \\
\hline Complete response, $\mathrm{n}(\%)$ & $1(4.6)$ \\
Partial response, $\mathrm{n}(\%)$ & $7(31.8)$ \\
Stable disease, $\mathrm{n}(\%)$ & $12(54.5)$ \\
Progressive disease, $\mathrm{n}(\%)$ & $2(9.1)$ \\
ORR (\%, CR+PR) & 36.4 \\
DCR $(\%, \mathrm{CR}+\mathrm{PR}+\mathrm{SD})$ & 90.9 \\
Time to response(m) & \\
Media(range) & $3.9(1.6,7.7)$ \\
Duration of response(m) & 6.8 \\
Media & $4 / 8(50.0)$ \\
ongoing, $\mathrm{n} / \mathrm{N}(\%)$ & \\
PFS(m) & $6.8(3.4,9.8)$ \\
Media $(95 \% \mathrm{Cl})$ & $17.3(16.1,18.5)$ \\
OS(m) & \\
Media (95\% Cl) &
\end{tabular}

CR, complete response; PR, partial response; $S D$, stable disease; PD, Progressive disease; ORR, objective response rate; $D C R$, disease control rate.

extra 1.4 months PFS and 7.7 months OS benefit compared with anlotinib alone. In comparison with sole PD-1 mAb treatment, the combination treatment also provided survival benefit. The median OS of nivolumab ranged from 9.2 months to 12.2 months in CheckMate 017 and CheckMate 057 clinical trials; 12.7 months of pembrolizumab in Keynote 010 clinical trial; for atezolizumab, the median OS ranged from 12.6 months to 13.8 months in OAK (third line therapy) and POPLAR clinical trial $(6,17)$. Therefore, the combination treatment gained an encouraging OS. This favorable OS was achieved for several reasons. The different treatment lines between Checkmate017, Checkmate057 (second-line) and our cohort (third line) were part of the reason for the prolonged OS, meanwhile, in standard third line treatment, anlotinib alone gained a favorable survival time, $\mathrm{PD}-1 \mathrm{mAb}$ also prolonged the survival time compared with chemotherapy. Besides, the adverse events were tolerable for patients with combination treatment, few patients withdrew the clinical trial due to the TRAEs. With no doubt, the combination of PD-1 mAb and anlotinib can gained a longer survival benefit via a synergistic way. The mechanism may be that anlotinib altered the tumor immune microenvironment and PD-1 $\mathrm{mAb}$ improved the vessel normalization, which leads to the mutual sensitization between these two drugs (15). For instance, anlotinib

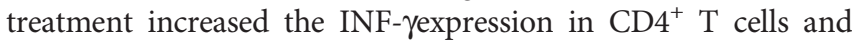
upregulated the tumor-infiltrating NK cells (11). Besides, anlotinib can inhibit PD-L1 expression on vascular endothelial cells so as to break through "immune tolerance barrier", it also promotes $\mathrm{CD} 8^{+} \mathrm{T}$ cell infiltration and improves the balance of CD8/Foxp3 (10). Many pro-angiogenic factors are derived from immune cells, such as M2like TAMs, immature dendritic cells, myeloid-derived suppressor cells (MDSCs), Trges and so on. These cells play various roles in the regulation of tumor angiogenesis (18). PD-1 mAb can suppress the activity of the immunosuppressive cells, indirectly down-regulate the angiogenic factors, and alleviate the abnormalities of tumor vessel $(19,20)$. In line with this, a number of experimental studies have confirmed that anti-angiogenesis agents combined with PD-1 mAb can reduce the tumor volume of tumor bearing mice $(21,22)$. 
A

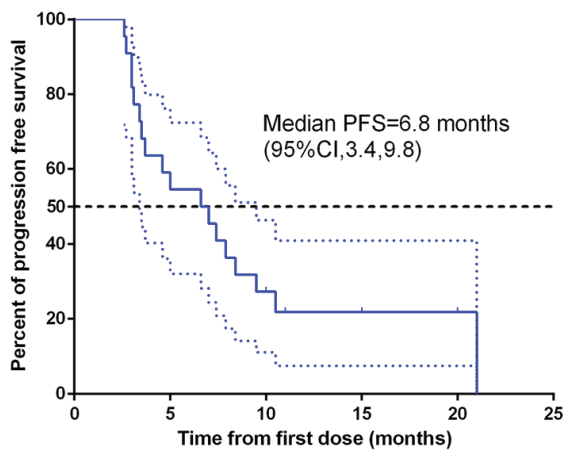

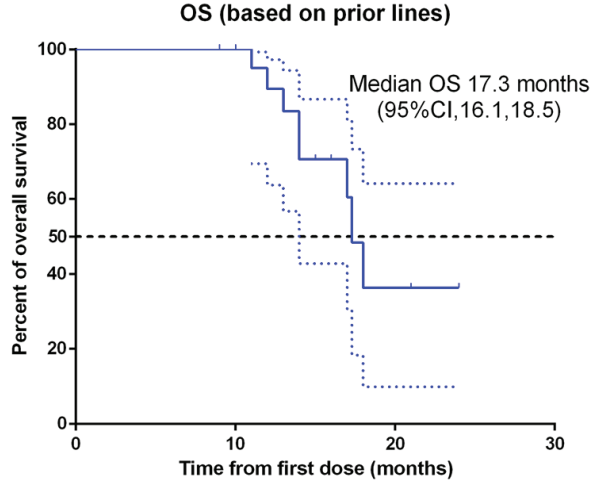

FIGURE 2 | Kaplan-Meier survival curve of progression-free survival (A) and overall survival (B) in 22 non-small cell lung cancer patients. PFS, progression-free survival; OS, over survival; $\mathrm{Cl}$, confidence interval.

A

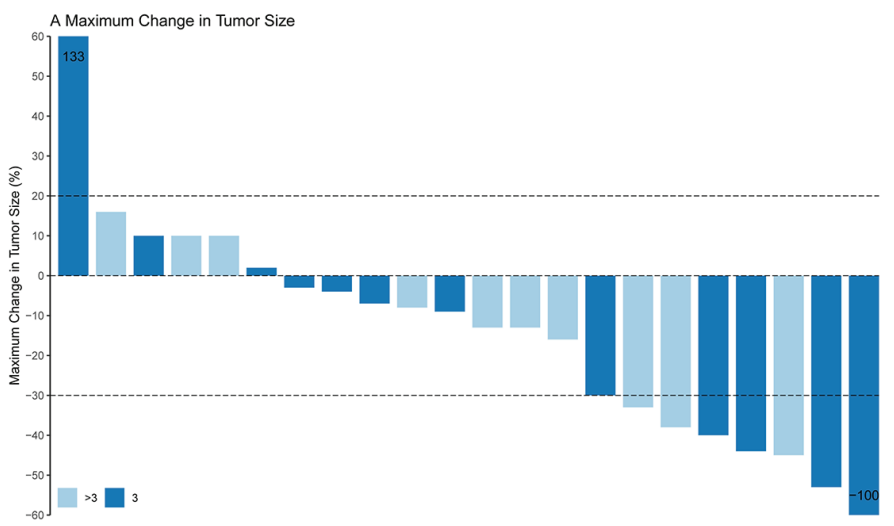

B

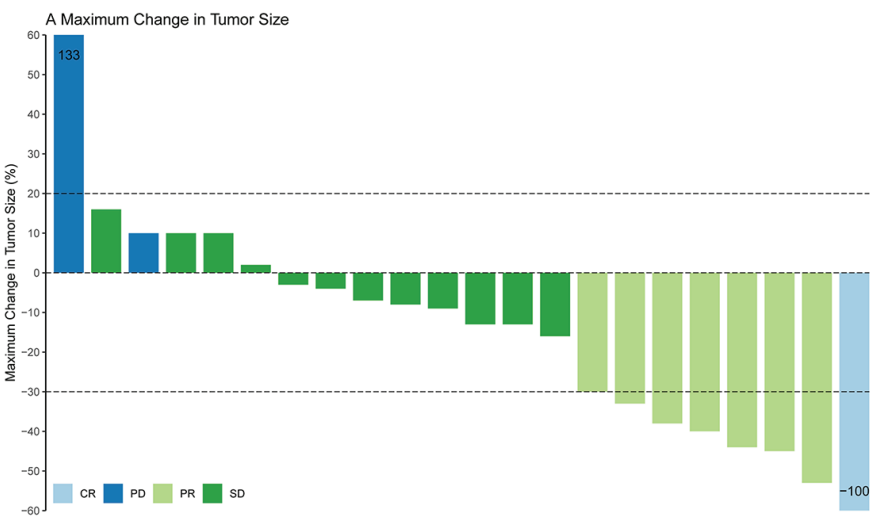

FIGURE 3 | Maximum change in tumor size based on the treatment lines (A) and tumor response (B) in 22 non-small cell lung cancer patients. CR, complete response; PR, partial response; SD, stable disease; PD, Progressive disease; ORR, objective response rate.

The median time to response (TTR) and the duration of response (DOR) of pembrolizumab in KEYNOTE 001 was 2.1 months and 18 months, respectively, and 101/495 (20.4\%) patients in this clinical trial were treatment naïve (23). In camrelizumab (PD-1 mAb) combined with apatinib treatment, the TTR was 3.7 months and DOR was 5.3 months (24). While in our study, the TTR was 3.9 months, which is longer than the results of both above studies, probably because that most patients in this study were third line or above line and 63.6\% (14/22) patients were with three or more organ metastases. Although the TTR was longer in our study, however, the DOR of our study is 6.8 months, which was lengthened than the camrelizumab 


\section{Anlotinib+PD-1(N=22)}

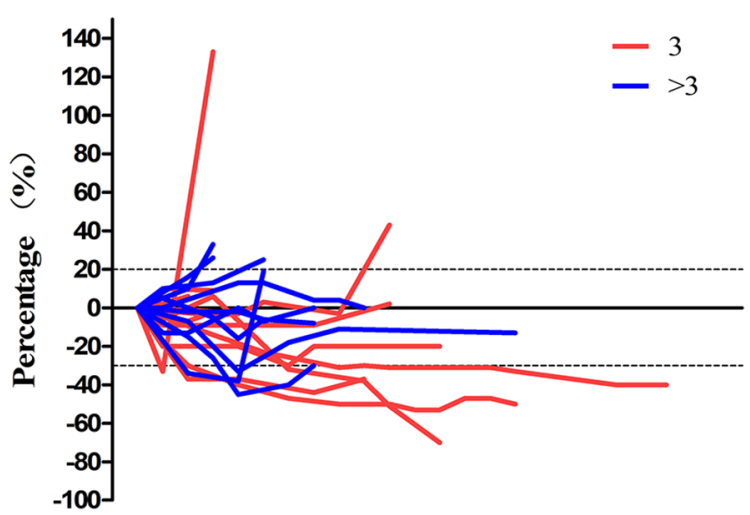

FIGURE 4 | The percent change in the sum of the longest diameter of target lesions diameter from the baseline in 22 non-small cell lung cancer patients.

combined with apatinib treatment. The results indicated that anlotinib combined with PD-1 mAb could also achieve longterm benefits.

It is worth noting that one patient achieved a CR response and had PFS for more than 20 months. The patient had complex mixed mutations, including K-RAS exon 2 missense mutation, TP53 exon 9 frameshift mutation, TAPBPL (TAP binding protein like) exon 2 missense mutation and SMARCA4 (SEI/SNF related, matrix associated, actin dependent regulator of chromatin, subfamily a, member 4) exon 20 missense mutation. Consistent with our findings, In Keynote 001, the median PFS of patients with K-RAS mutation and TP53 mutation treated with pembrolizumab was 14.7 months, much higher than that of 3.5 months in the wild-type group of K-RAS (25). Interestingly, it has been reported that a squamous-cell NSCLC patient with TP53 and KRAS co-mutation was treated with pembrolizumab combined with gemcitabine. The

TABLE 3 | Treatment-related adverse events.

\begin{tabular}{lcc}
\hline AEs & $\begin{array}{c}\text { Grade 1-2, } \mathbf{n} \\
\mathbf{( \% )}\end{array}$ & $\begin{array}{c}\text { Grade 3-4, } \mathbf{~} \\
\mathbf{( \% )}\end{array}$ \\
\hline Fatigue & $10(45.5)$ & 0 \\
Decreased appetite & $9(40.9)$ & 0 \\
Nausea & $3(13.6)$ & 0 \\
Weight decrease & $4(18.2)$ & 0 \\
Rash & $8(36.4)$ & $1(4.6)$ \\
Hypertension & $10(45.5)$ & $2(9.1)$ \\
Diarrhea & $7(31.8)$ & $1(4.6)$ \\
Mouth ulceration & $5(22.7)$ & $2(9.1)$ \\
Dysphonia & $4(18.2)$ & 0 \\
Pneumonitis & $4(18.2)$ & $1(4.6)$ \\
Cough & $2(9.1)$ & 0 \\
Hepatic function abnormal & $3(13.6)$ & 0 \\
Hypothyroidism & $4(18.2)$ & 0 \\
proteinuria & $5(22.7)$ & 0 \\
Palmar-plantar erythrodysaesthesia & $4(18.2)$ & 0 \\
syndrome & &
\end{tabular}

AEs, adverse events. therapeutic effect reached PR and PFS was more than 7 months (26). The K-RAS mutation status may be an indicator of good response to the PD-1 mAb, the TP53 mutation patients also showed good response to anlotinib (27). So clinical trial like combination of anlotinib and PD-1 mAb may be promising in patients with K-RAS mutation and TP53 mutation co-existed. In addition, recently, it is generally believed that PD-L1 high expression is correlated with good prognosis in patients treated with PD-1 mAb $(28,29)$. In keynote 024 , the patients whose PD-L1 TPS score $>50 \%$ treated with pembrolizumab gained a median OS of 30 months (30), and in keynote-042 (TPS $>50 \%$ Subgroup), the median OS is 20 months (31). It is impressive that in keynote 024, three patients treated with single pembrolizumab achieved CR $(30,32)$. In our study the CR patient also had a PD-L1 TPS above 50\%. Therefore, among the three factors of K-RAS mutation, TP53 mutation and high score of PD-L1 TPS, which was the real prognostic factor is still uncertain. More clinical trials or experience should be focused on the factors.

For patients with brain metastasis, the prognosis was worse than the patients without brain metastasis, but the only 22 patients was included in our cohort, it may not be convincing enough until more prospective studies were conducted.

The adverse events of the combination treatment were tolerable in our patients, so the combination was safe and could be popularized in the future. The most common grade 1-2 adverse events were hypertension and fatigue (33), which were in line with the ALTER-0303 clinical trial. However, the thyroid stimulating hormone (TSH) elevation and hypertriglyceridemia were less common in our center, which might due to a limited number of cases. The common adverse events of PD-1 mAb were diarrhea, rash, decreased appetite, nausea, anemia and neutropenia (34). The organ specific immune-related adverse events were hypothyroidism, pneumonitis, colitis, hepatitis and hypophysitis (35). In our study, four (18.2\%) patients had mild hypothyroidism, and four (18.2\%) patients had grades 1-2 pneumonitis. These adverse events were consistent with the previous clinical trial. However, it should be noted that the median time to follow up was 384 days (95\%CI: 298 days, 469 days), some immune related adverse event can occur later, for example, the hepatitis can occur after 34 weeks exposed to nivolumab treatment (36). The median time to onset of late-immune-related adverse events was 16.6 months in a multi-center study (37). So the immune-related adverse events need to follow up later.

Our study has obvious limitations because only 22 patients were included, resulting in the inability to carry out univariate or multivariate analysis. The anlotinib dose, different pathology types, number of metastasis organs, treatment lines and other factors may affect the efficacy of combination treatment. More clinical trials or clinical experience are needed to identify the beneficial patient group. In addition, retrospective study may lose some detailed information of the patients, like the gene mutation and PD-L1 of some patients were unknown; besides, multi PD-1 $\mathrm{mAb}$ were used in our center, which might produce diverse efficacy. In this study, three patients with EGFR mutation had a history of target therapy, the PFS of three patients were very different $(3,5$, and 10.5 months, respectively). So we cannot come to a conclusion right now, this requires more data based on these subtype of patients. To 
sum up, our retrospective analysis shows that the efficacy and safety of the combination therapy of anlotinib and PD-1 mAb are encouraging and worthy of further clinical trials.

\section{DATA AVAILABILITY STATEMENT}

The raw data supporting the conclusions of this article will be made available by the authors, without undue reservation.

\section{ETHICS STATEMENT}

Written informed consent was obtained from the individual(s), and minor(s)' legal guardian/next of kin, for the publication of any potentially identifiable images or data included in this article.

\section{AUTHOR CONTRIBUTIONS}

CZ: Data curation, Methodology, Investigation. XZ: Data curation, Methodology, Writing original draft. LR: Data curation, Writing-review and editing. LY: Data curation,

\section{REFERENCES}

1. Herbst RS, Morgensztern D, Boshoff C. The biology and management of nonsmall cell lung cancer. Nature (2018) 553(7689):446-54. doi: 10.1038/ nature 25183

2. Ramalingam SS, Vansteenkiste J, Planchard D, Cho BC, Gray JE, Ohe Y, et al. Overall Survival with Osimertinib in Untreated, EGFR-Mutated Advanced NSCLC. N Engl J Med (2020) 382(1):41-50. doi: 10.1056/NEJMoa1913662

3. Borghaei H, Paz-Ares L, Horn L, Spigel DR, Steins M, Ready NE, et al. Nivolumab versus Docetaxel in Advanced Nonsquamous Non-Small-Cell Lung Cancer. N Engl J Med (2015) 373(17):1627-39. doi: 10.1056/ NEJMoa1507643

4. Doroshow DB, Sanmamed MF, Hastings K, Politi K, Rimm DL, Chen L, et al. Immunotherapy in Non-Small Cell Lung Cancer: Facts and Hopes. Clin Cancer Res (2019) 25(15):4592-602. doi: 10.1158/1078-0432.CCR-18-1538

5. Rizvi NA, Mazières J, Planchard D, Stinchcombe TE, Dy GK, Antonia SJ, et al. Activity and safety of nivolumab, an anti-PD-1 immune checkpoint inhibitor, for patients with advanced, refractory squamous non-small-cell lung cancer (CheckMate 063): a phase 2, single-arm trial. Lancet Oncol (2015) 16(3):257-65. doi: 10.1016/S1470-2045(15)70054-9

6. Rittmeyer A, Barlesi F, Waterkamp D, Park K, Ciardiello F, von PJ, et al. Atezolizumab versus docetaxel in patients with previously treated non-smallcell lung cancer (OAK): a phase 3, open-label, multicentre randomised controlled trial. Lancet (2017) 389(10066):255-65. doi: 10.1016/S0140-6736 (16)32517-X

7. Sheng Z, Zhu X, Sun Y, Zhang Y. The efficacy of anti-PD-1/PD-L1 therapy and its comparison with EGFR-TKIs for advanced non-small-cell lung cancer. Oncotarget (2017) 8(34):57826-35. doi: 10.18632/oncotarget.18406

8. Kim JY, Lee KH, Kang J, Borcoman E, Saada-Bouzid E, Kronbichler A, et al. Hyperprogressive Disease during Anti-PD-1 (PDCD1) / PD-L1 (CD274) Therapy: A Systematic Review and Meta-Analysis. Cancers (Basel) (2019) 11 (11):1699. doi: 10.3390/cancers11111699

9. Han B, Li K, Wang Q, Zhang L, Shi J, Wang Z, et al. Effect of Anlotinib as a ThirdLine or Further Treatment on Overall Survival of Patients With Advanced NonSmall Cell Lung Cancer: The ALTER 0303 Phase 3 Randomized Clinical Trial. JAMA Oncol (2018) 4(11):1569-75. doi: 10.1001/jamaoncol.2018.3039
Methodology. QP: Methodology, Supervision. HP: Methodology, Supervision. WH: Methodology, Supervision, Writing-review and editing. All authors contributed to the article and approved the submitted version.

\section{FUNDING}

This work was supported by the National Natural Science Foundation of China (81972745 and 81703072), the Ten Thousand Plan Youth Talent Support Program of Zhejiang Province (ZJWR0108009), and the Zhejiang Medical Innovative Discipline Construction Project-2016, and the Hangzhou Health and Family Planning and Science and Technology Program (OO20190347).

\section{SUPPLEMENTARY MATERIAL}

The Supplementary Material for this article can be found online at: https://www.frontiersin.org/articles/10.3389/fonc.2020.619010/ full\#supplementary-material.

10. Liu S, Qin T, Liu Z, Wang J, Jia Y, Feng Y, et al. anlotinib alters tumor immune microenvironment by downregulating PD-L1 expression on vascular endothelial cells. Cell Death Dis (2020) 11(5):309. doi: 10.1038/s41419-0202511-3

11. Yang Y, Li L, Jiang Z, Wang B, Pan Z. Anlotinib optimizes anti-tumor innate immunity to potentiate the therapeutic effect of PD-1 blockade in lung cancer. Cancer Immunol Immunother (2020) 69(12):2523-32. doi: 10.1007/s00262020-02641-5

12. Zhang Y, Zhao M, Cao S, Zhang X, Du Y. Unexpected favorable outcome to sintilimab plus bevacizumab in an EGFR-mutated non-small cell lung cancer patient: A case report. Thorac Cancer (2020) 11(9):2717-22. doi: 10.1111/ 1759-7714.13569

13. Han TCB, Zhong R. Efficacy and safety of Sinitilimab with Anlotinib as firstline therapy for advanced Non-Small Cell Lung Cancer(NSCLC). J Thorac Oncol (2019) 14(10):S439. doi: 10.1016/j.jtho.2019.08.269

14. Cai X, Wei B, Li L, Chen X, Liu W, Cui J, et al. Apatinib enhanced anti-PD-1 therapy for colon cancer in mice via promoting PD-L1 expression. Int Immunopharmacol (2020) 88:106858. doi: 10.1016/j.intimp.2020.106858

15. Allen E, Jabouille A, Rivera LB, Lodewijckx I, Missiaen R, Steri V, et al. Combined antiangiogenic and anti-PD-L1 therapy stimulates tumor immunity through HEV formation. Sci Transl Med (2017) 9(385):eaak9679. doi: 10.1126/scitranslmed.aak9679

16. Lanitis E, Irving M, Coukos G. Targeting the tumor vasculature to enhance T cell activity, Curr. Opin Immunol (2015) 33:55-63. doi: 10.1016/j.coi.2015.01.011

17. Fehrenbacher L, Spira A, Ballinger M, Kowanetz M, Vansteenkiste J, Mazieres $\mathrm{J}$, et al. Atezolizumab versus docetaxel for patients with previously treated non-small-cell lung cancer (POPLAR): a multicentre, open-label, phase 2 randomised controlled trial. Lancet (2016) 387(10030):1837-46. doi: 10.1016/ S0140-6736(16)00587-0

18. Lee WS, Yang H, Chon HJ, Kim C. Combination of therapy and immune checkpoint blockade normalizes vascular-immune crosstalk to potentiate cancer immunity. Exp Mol Med (2020) 52(9):1475-85. doi: 10.1038/s12276020-00500-y

19. Huang Y, BYS K, Chan CK, Hahn SM, Weissman IL, Jiang W. Improving immune-vascular crosstalk for cancer immunotherapy. Nat Rev Immunol (2018) 18(3):195-203. doi: 10.1038/nri.2017.145 
20. Rahma OE, Hodi FS. The Intersection between Tumor Angiogenesis and Immune Suppression. Clin Cancer Res (2019) 25(18):5449-57. doi: 10.1158/ 1078-0432.CCR-18-1543

21. Zhao S, Ren S, Jiang T, Zhu B, Li X, Zhao C, et al. Low-Dose Apatinib Optimizes Tumor Microenvironment and Potentiates Antitumor Effect of PD-1/PD-L1 Blockade in Lung Cancer. Cancer Immunol Res (2019) 7(4):63043. doi: 10.1158/2326-6066.CIR-17-0640

22. Schmittnaegel M, Rigamonti N, Kadioglu E, Cassará A, Wyser RC, Kiialainen A, et al. Dual angiopoietin-2 and VEGFA inhibition elicits antitumor immunity that is enhanced by PD-1 checkpoint blockade. Sci Transl Med (2017) 9(385):eaak9670. doi: 10.1126/scitranslmed.aak9670

23. Garon EB, Hellmann MD, Rizvi NA, Carcereny E, Leighl NB, Ahn MJ, et al. Five-Year Overall Survival for Patients With Advanced Non-Small-Cell Lung Cancer Treated With Pembrolizumab: Results From the Phase I KEYNOTE001 Study. J Clin Oncol (2019) 37(28):2518-27. doi: 10.1200/JCO.19.00934

24. Zhou C, Gao G, Wang YN, Zhao J, Chen G, Liu Z, et al. Efficacy of PD-1 monoclonal antibody SHR-1210 plus apatinib in patients with advanced nonsquamous NSCLC with wild-type EGFR and ALK. J Clin Oncol (2019) 37(15_suppl):9112-9112.

25. Dong ZY, Zhong WZ, Zhang XC, Su J, Xie Z, Liu SY, et al. Potential Predictive Value of TP53 and KRAS Mutation Status for Response to PD-1 Blockade Immunotherapy in Lung Adenocarcinoma. Clin Cancer Res (2017) 23 (12):3012-24. doi: 10.1016/j.jtho.2016.11.504

26. Fang C, Zhang C, Zhao WQ, Hu WW, Wu J, Ji M. Co-mutations of TP53 and KRAS serve as potential biomarkers for immune checkpoint blockade in squamous-cell non-small cell lung cancer: a case report. BMC Med Genomics (2019) 12(1):136. doi: 10.1186/s12920-019-0592-6

27. Fang S, Cheng W, Zhang M, Yang R. Association of TP53 Mutations with Response to Anlotinib Treatment in Advanced Non-Small Cell Lung Cancer. Onco Targets Ther (2020) 13:6645-50. doi: 10.2147/OTT.S257052

28. Lin H, Wei S, Hurt EM, Green MD, Zhao L, Vatan L, et al. Host expression of PD-L1 determines efficacy of PD-L1 pathway blockade-mediated tumor regression. J Clin Invest (2018) 128(4):1708. doi: 10.1172/JCI120803

29. Shen X, Zhao B. Efficacy of PD-1 or PD-L1 inhibitors and PD-L1 expression status in cancer: meta-analysis. BMJ (2018) 362:k3529. doi: 10.1136/bmj.k3529

30. Reck M, Rodríguez-Abreu D, Robinson AG, Hui R, Csőszi T, Fülöp A, et al. Updated Analysis of KEYNOTE-024: Pembrolizumab Versus Platinum-Based Chemotherapy for Advanced Non-Small-Cell Lung Cancer With PD-L1
Tumor Proportion Score of 50\% or Greater. J Clin Oncol (2019) 37(7):53746. doi: 10.1200/JCO.18.00149

31. TSK M, Wu YL, Kudaba I, Kowalski DM, Cho BC, Turna HZ, et al. Pembrolizumab versus chemotherapy for previously untreated, PD-L1expressing, locally advanced or metastatic non-small-cell lung cancer (KEYNOTE-042): a randomised, open-label, controlled, phase 3 trial. Lancet (2019) 393(10183):1819-30. doi: 10.1016/S0140-6736(18)32409-7

32. Reck M, Rodríguez-Abreu D, Robinson AG, Hui R, Csőszi T, Fülöp A, et al. Pembrolizumab versus Chemotherapy for PD-L1-Positive Non-Small-Cell Lung Cancer. N Engl J Med (2016) 375(19):1823-33. doi: 10.1056/NEJMoa1606774

33. Si X, Zhang L, Wang H, Zhang X, Wang M, Han B, et al. Management of anlotinib-related adverse events in patients with advanced non-small cell lung cancer: Experiences in ALTER-0303. Thorac Cancer (2019) 10(3):551-6. doi: 10.1111/1759-7714.12977

34. Hellmann MD, Paz-Ares L, Bernabe CR, Zurawski B, Kim SW, Carcereny CE, et al. Nivolumab plus Ipilimumab in Advanced Non-Small-Cell Lung Cancer. N Engl J Med (2019) 381(21):2020-31. doi: 10.1056/NEJMoa1910231

35. Baxi S, Yang A, Gennarelli RL, Khan N, Wang Z, Boyce L, et al. Immunerelated adverse events for anti-PD-1 and anti-PD-L1 drugs: systematic review and meta-analysis. BMJ (2018) 360:k793. doi: 10.1136/bmj.k793

36. Imafuku K, Yoshino K, Yamaguchi K, Tsuboi S, Ohara K, Hata $H$. Successful Treatment of Sudden Hepatitis Induced by Long-Term Nivolumab Administration. Case Rep Oncol (2017) 10(1):368-71. doi: 10.1159/000471480

37. Nigro O, Pinotti G, De Galitiis F, Di Pietro FR, Giusti R, Filetti M, et al. Late immune-related adverse events in long-term responders to $\mathrm{PD}-1 / \mathrm{PD}-\mathrm{L} 1$ checkpoint inhibitors: A multicentre study. Eur J Cancer (2020) 134:19-28. doi: 10.1093/annonc/mdz449.009

Conflict of Interest: The authors declare that the research was conducted in the absence of any commercial or financial relationships that could be construed as a potential conflict of interest.

Copyright (c) 2021 Zhai, Zhang, Ren, You, Pan, Pan and Han. This is an open-access article distributed under the terms of the Creative Commons Attribution License (CC BY). The use, distribution or reproduction in other forums is permitted, provided the original author(s) and the copyright owner(s) are credited and that the original publication in this journal is cited, in accordance with accepted academic practice. No use, distribution or reproduction is permitted which does not comply with these terms. 